\title{
CT-guided Interventional Therapy of Back Pain - Predictors of Success in Treatment
}

\section{CT-gesteuerte lumbale Schmerztherapie - Kann die Verteilung des Medikaments den Erfolg der Behandlung voraussagen?}

Authors

Christoph Alexander Stueckle ${ }^{1,2,4}$, Sarah Talarczyk ${ }^{1,4}$, Benedikt Hackert ${ }^{3}$, Martin Wawro ${ }^{4}$, Patrick Haage ${ }^{2}$, Ulrich Weger ${ }^{3}$

Affiliations

1 University Witten/Herdecke, Faculty of Health, Witten, Germany

2 Diagnostic and Interventional Radiology, HELIOS University Hospital Wuppertal, University Witten/ Herdecke, Germany

3 Department of Psychology/ Psychotherapy, University Witten/Herdecke, Germany

4 Medical Center MVZ Professor Uhlenbrock \& Partner $\mathrm{GmbH}$, Dortmund, Germany

Key words

periradicular pain therapy, back pain, CT guided intervention, pain therapy, spine

received 01.02 .2020

accepted 11.04.2020

published online 16.07.2020

Bibliography

Fortschr Röntgenstr 2021; 193: 42-48

DOI 10.1055/a-1161-9358

ISSN 1438-9029

(c) 2020. Thieme. All rights reserved.

Georg Thieme Verlag KG, Rüdigerstraße 14,

70469 Stuttgart, Germany

Correspondence

Dr. Christoph Alexander Stueckle

Fakultät für Gesundheit

Universität Witten/Herdecke

MVZ Prof. Uhlenbrock, Glückaufstraße 10,

44575 Castrop-Rauxel, Germany

Tel.: + 49 231/94331413

c.stueckle@mvz-uhlenbrock.de

\section{ABSTRACT}

Purpose The aim of the presented study was to investigate if distribution of the drug-contrast medium mixture in $\mathrm{CT}$-guided periradicular therapy can predict intervention success.

Materials and Methods Over a 15-month period, 97 patients admitted to our institution by a pain physician for CT guided periradicular therapy were treated. In 420 CT guided interventions, we measured the relative distribution of the drug mixture in relationship to the neuroforamen. The distribution was correlated to the patient's pain score over time. In addition, dependence of success in treatment with regards to the treating physician was evaluated.

Results Ninety percent of the patients experienced a decrease of pain score under therapy, only $10 \%$ showed no change. On average there was an improvement in pain score from $7.4(2-10)$ before therapy to $3.7(0-9)$ at the end of therapy. We observed a weak correlation of $-0.22(p<0.028)$ between distribution of drug mixture to the neuroforamen and reduction of pain score. The therapy outcome displayed a dependency regarding the treating physician $(p<0.0084)$.

Conclusion CT guided periradicular therapy offers good pain reduction in most of the patients. Drug distribution near the affected nerve and treating physician are factors for clinical success.

Key Points:

- CT-guided periradicular therapy shows a good or very good improvement in the symptoms in $90 \%$ of the treated patients.

- In addition to technical performance, the treating radiologist is an important parameter determining success.

- The success of CT-guided treatment is only moderately correlated with an absolute distribution of the drug mixture.

\section{Citation Format}

- Stueckle CA, Talarczyk S, Hackert B et al. CT-guided Interventional Therapy of Back Pain - Predictors of Success in Treatment. Fortschr Röntgenstr 2021; 193: 42-48

\section{ZUSAMMENFASSUNG}

Ziel Die vorliegende Arbeit untersucht, ob über die Verteilung des Medikamenten-Kontrastmittel-Gemisches im Rahmen der CT-gesteuerten periradikulären Therapie eine Aussage über den zu erwartenden Erfolg getroffen werden kann.

Material und Methode Über einen Zeitraum von 15 Monaten wurden konsekutiv insgesamt 97 Patienten untersucht, die vom Schmerztherapeuten zur CT-gesteuerten periradikulären Therapie überwiesen worden sind. Insgesamt konnten wir 420 CT-gesteuerte Interventionen einschließen. Wir haben die relative Verteilung des Medikamentengemisches 
in Korrelation zum Neuroforamen mit dem Schmerzscore des Patienten im Verlauf korreliert. Zudem wurde überprüft, ob es eine Abhängigkeit des Behandlungserfolgs vom behandelnden Arzt gibt.

Ergebnisse Die Patienten zeigten insgesamt ein sehr gutes Outcome: $90 \%$ der mittels CT-gesteuerter periradikulärer Therapie behandelten Patienten zeigten eine Verbesserung der Schmerzsymptomatik. $10 \%$ zeigten keine Verbesserung durch die CT-gestützte Intervention. Im Durchschnitt verbesserte sich der Schmerzscore von 7,4 (2-10) vor der Intervention auf 3,7 (0-9) am Ende der Therapie. Es zeigte sich eine negative Korrelation von $-0,22(p<0,028)$ zwischen der Schmerzreduktion und der Nähe des Medikamentengemisches zum Neuroforamen. Zudem zeigten unsere Daten eine Abhängigkeit des Outcomes vom behandelnden Arzt ( $p<0,0084)$.

Schlussfolgerungen Die CT-gesteuerte periradikuläre Therapie führt in den meisten Fällen zu einer guten Schmerzreduktion. Neben einer guten Platzierung des Medikamentengemisches in der Nähe der affektierten Nervenwurzel scheint auch der ausführende Arzt eine Rolle beim Outcome für den Patienten zu spielen.

\section{Introduction}

Back pain is one of the most common medical and economic issues among industrialized countries, with a lifetime prevalence of 20 to $40 \%$ [1, 2]. Back pain leads to incapacity to work as well as numerous therapies and various therapeutic attempts. In addition, due to loss of manpower, it causes enormous costs in the health care system and the economy [2, 3]. Studies put the cost of back pain at $3-10 \%$ of the gross domestic product [4]. The costs are divided roughly equally between direct costs for the health care system and indirect costs for other service providers such as pension and social security funds [4].

Back pain often goes away spontaneously; various studies show that in $23-48 \%$ of patients with disc-related back pain the discomfort disappears spontaneously and without therapy after six to eight weeks. However, at the same time, a probability of recurrence of the complaint within one year is given as $24-80 \%$ [1, 5-7]. There are many types of therapy available, which are used in very different forms and often depend on the treating physician. On the one hand, there are conservative measures such as physiotherapy, pharmacological therapy and acupuncture, CT- and fluoroscopy-guided interventional procedures as well as psychotherapeutic and behavioral therapy approaches; on the other hand - in case of failure of conservative forms of therapy - various surgical procedures are available [3, 6].

In recent years, $\mathrm{CT}$-guided therapy has shown good results with very few side effects for both discogenic and osseous-discogenic back pain [7-9]. Various studies report an improvement in pain symptoms after CT-guided interventional therapy in 52-75\% of patients $[1,7,10]$.

Depending on the cause of pain, CT-guided pain therapy can use various approaches. In the case of wear of the facet joints, therapy of the joint is correspondingly performed, with the target point being the joint itself. Interlaminar epidural therapy is usually chosen in cases of medial discogenic narrowing of the spinal canal. For neuroforaminal constriction - whether discogenic or combined osseous-discogenic - transforaminal epidural or peridural therapy is preferred $[1,11,12]$. CT-guided periradicular therapy is common because - among other things - it has a high success rate (52-90\%) with few side effects and low costs [12-16].
Nevertheless, it is currently completely unclear which factors ultimately determine the success of CT-guided interventional pain therapy for specific back pain. Published studies and metaanalyses show a wide range of results as well as the types of intervention and modifications. Finally, to the best of our knowledge, it has not yet been investigated whether technical success is decisive for patient outcome or whether other factors besides good technical performance also influence treatment success.

\section{Goals}

The aim of this study is to determine whether the measurable technical success of CT-guided periradicular therapy correlates positively with a reduction of vertebrogenic pain and whether there is a correlation with the treating physician.

\section{Materials and Methods}

The prospective study was conducted between November 2016 and June 2018 following a positive vote of the Ethics Committee of the University of BLINDED. Included were all patients who were sent to our institute by their pain therapist for CT-guided periradicular therapy in the area of the lumbar spine. Before inclusion in the study, patients were informed in detail about its purpose and benefits and gave their written consent. Only patients with imaging performed within six weeks prior to the study were included, in which a clear morphological change matching the symptoms could be demonstrated. Only adult patients were included, $61 \%$ of whom had a discogenic nerve root affection, $3 \%$ an exclusively osseous nerve root affection and $36 \%$ a combination of both. Among the patients with a discogenic nerve root affection there was direct contact between the herniated disc and the nerve in $59 \%$ of the cases, and in $41 \%$ there was a displacement or deformation of the nerve due to contact with the herniated disc. In patients with combined osseous-discogenic stenosis, the distribution was $34 \%$ of cases directly contacting the nerve root and in $66 \%$ there was dislocation or deformation, in the cases with exclusively osseous constriction $54 \%$ and $46 \%$. In conjunction with the referring pain therapy physicians, the image morphological findings were clearly identified with the symptoms in relation to the sensitive dermatome and the characteristic muscle, and in the 
case of multisegmental symptoms, the main segment was identified by means of experimental infiltration, which was then the only pain point to be evaluated in the study.

Patients who, during CT-guided periradicular therapy (PRT), simultaneously received further treatment by another physician were not included in the study. During the observation period, 97 of a total of 718 patients met the inclusion criteria and were willing to participate in the study. A total of 429 CT-guided interventions were evaluated, and 858 patient interviews were conducted. Participants included 53 female and 44 male patients with an average age of 56.9 years (range: 25 to 83 years).

At least 24 hours before the CT-guided intervention, a radiologist provided detailed information to each patient. The information session particularly covered explanations of the intervention, the risks as well as the expected benefits.

On the days of the CT-guided periradicular therapy, prior to the procedure, a psychologist surveyed the patients regarding their current pain. In the course of this interview, the patient had to identify current pain based on a visual, analog pain scale ranging from 0 (no pain) to 10 (maximum imaginable pain). To ensure that there was adequate anticipation of the pain scale, the research assistant explained the scale: 0: "Everything is great, well-being unaffected by pain, feel like a kid". 10: "Maximum pain, feels like a knife moving around in my back, it couldn't be worse".

During the procedure, the patients were placed in prone position. The intervention was planned on a short, lateral topogram at the level of the previously identified intervention height, and a CT slice or short spiral was performed to plan the procedure. After careful local disinfection, sterile preparation and marking of the puncture site, the procedure was performed under CT guidance with low-dose technique using CareDose 4D. According to the instructions of the performing radiologist, the needle position was checked during the procedure and the distribution of the drug mixture was documented. The interventions were performed either on a Siemens Emotion 6 or Siemens Definition 64.

Depending on the patient's body mass, a 22G needle with a length of $90 \mathrm{~mm}$ or $120 \mathrm{~mm}$ was used (Becton Dickinson SA, S. Agustin del Gualdix, Spain). As local anesthetic, $2 \%$ Meaverin (Meaverin 2\%, Puren Pharma, Munich, Germany) was employed. lohexol (Accupaque 240, GE Healthcare, Munich, Germany) was used as a contrast medium to document the distribution of the drug mixture, and $20 \mathrm{mg}$ triamcinolone was administered as the corticoid (Volon A, 2x10 mg, Dermapharm AG, Gruenwald, Germany). After successful intervention, the patients spent between 30 and 60 minutes in our institute to rule out acute side effects of the therapy. After the intervention, the patients were again briefly seen by the performing physician to ascertain that no side effects occurred and to send the patient home.

The time window between the individual PRT treatments was on average five days (range: one to 14 days). Prior to each new PRT examination, the patient was interviewed by a psychologist regarding the current pain situation and well-being. In the course of this investigation, the current pain score was again assessed based on the 10-part visual pain scale.

The CT-guided intervention was performed randomly by one of five radiologists, comprising two female and three male physi- cians. The most experienced colleague has a total of 18 years, the least experienced two years experience in interventional pain therapy.

Each time after the CT-guided procedure, the patient was surveyed again by the research assistant regarding his current pain situation. The treatment was always terminated when the patient reported a complete or almost complete improvement of symptoms under therapy (pain score $\leq 1$ ), when five treatments had been carried out, or when the patient stopped the treatment prematurely.

The study compares the distribution of the drug mixture around the target nerve relative to the success of the therapy. Thus the question arises whether success is greater if a higher percentage of the drug mixture is localized in the immediate vicinity of the nerve compared to otherwise?

Anatomically, the emerging nerve root in the area of the lumbar spine is largely limited by bony structures, so that the available distribution space is severely restricted ( $\triangleright$ Fig. 1 ); thus, the applied drug mixture is distributed in an unpredictable manner ( $\vee$ Fig. 1). In order to make a reproducible and reliable statement about the distribution of the drug mixture around the nerve root, we have determined the radius in which at least $50 \%$ of the drug mixture is located. This distance was uniformly defined for the measurements as the main part of the drug deposit. The analysis was performed by our own software (developer: BLINDED), which, using a segmentation algorithm, determines the local contrast medium distribution around the nerve after marking the target nerve in the neural foramen. The reference point for the software is the center of the neural foramen. The results were reviewed by an experienced radiologist (BLINDED).

We divided the changes in the pain scores during therapy into three categories: reduction by less than 0.25 points $=$ no success. Reduction of the pain score between 0.25 and $3.0=$ good result. Reduction of the pain score by more than 3.0 points = very good result. In the event a patient initially gave a very low pre-therapeutic pain score $(<3)$, we also rated freedom from symptoms as a very good result.

Statistical analysis of the examination data was performed using a database created in MatLab R2017b (MathWorks, Natick, Massachusetts, USA).

\section{Results}

On average, 4.4 treatments were performed per patient (range: one to five treatments) with an average treatment time of 5.3 weeks (range: one to 18 weeks).

In a comparison of the pain score at the beginning of therapy with the pain score at the end of therapy, $90 \%$ of patients showed an improvement. Most patients (49\%) showed a very good improvement of pain symptoms, and $41 \%$ a good improvement of pain symptoms under therapy. Only $8 \%$ of the treated patients showed no improvement of symptoms under therapy. Worsening of symptoms occurred under therapy in $2 \%$ of patients.

A total of nine patients ended the treatment prematurely. Two patients stopped due to a worsening of their symptoms under therapy; three patients withdrew due to an already sufficient 


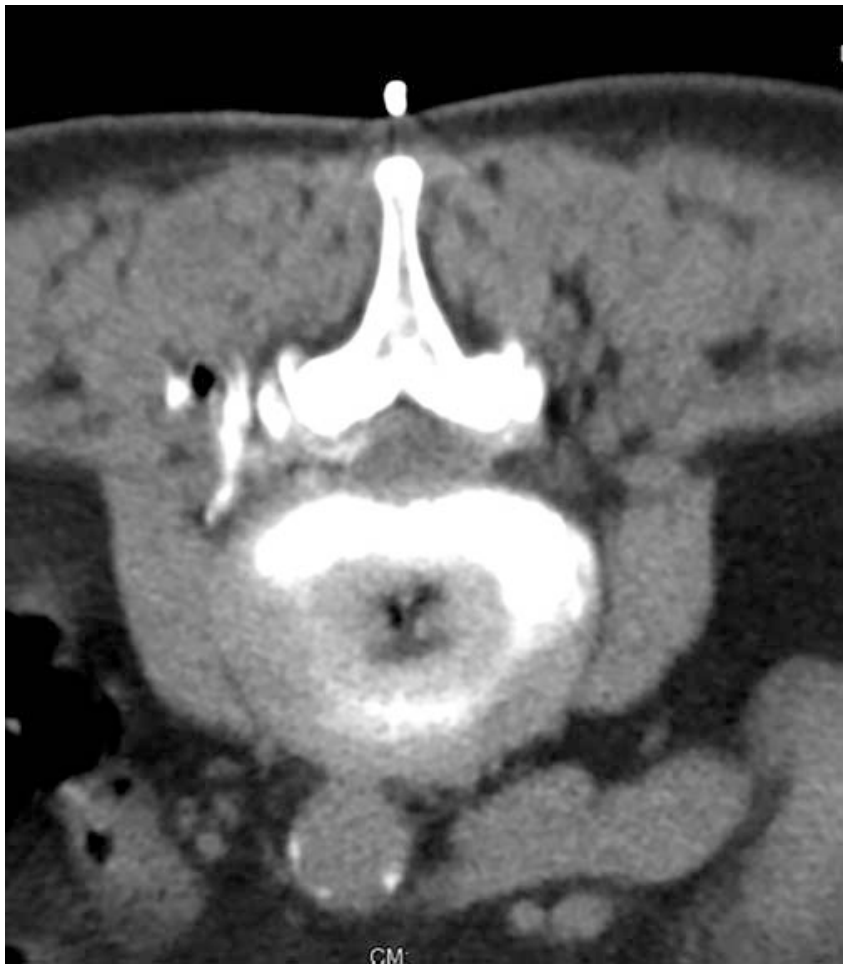

- Fig. 1 Distribution of the drug mixture after CT-guided periradicular therapy in segment L2/3. Only small portions are located in the neural foramen or intraspinally, the largest portions are extraforaminal.

improvement of their symptoms from their point of view; two patients halted the treatment without giving reasons; two patients terminated due to upcoming vacation.

The average pain score before therapy was 7.4 (range: 2-10), after therapy 3.7 (range: 0-9).

There was a weakly negative Spearman-rank correlation of $0.22(p<0.028)$ between the reduction of the pain score and the distance of the drug deposit. This means that the proximity of the majority of the drug to the nerve correlates with a decrease in the pain score ( $\nabla$ Fig. 2 ). In $85 \%$ there is direct contact between the contrast medium and the nerve root, in $15 \%$ no direct contact.

The distribution of the medication deposit as a distance to the target point was shown separately for each of the treating radiologists. In each case a shift of the maximum drug deposit to greater distances was observed, which is anatomically explainable, since the intraspinal and epidural distribution space is much smaller than the extraforaminal. Nevertheless, a measurable difference among the involved radiologists was observed ( $\mathbf{F i g . 3}$ ). Thus, in the totality of his performed therapies, the technically best radiologist was $5 \%$ better than average at distributing the drug mixture, while the technically worst radiologist was $6.5 \%$ worse than average at distributing the drug mixture. Overall, the differences in the distribution of the drug deposit were very small, ranging from 14.6 to $17.5 \mathrm{~mm}$.

If the success of the intervention, measured as the reduction of the pain score, is included in the analysis, Physician E was the most successful with a pain reduction of 1.3 points per intervention,

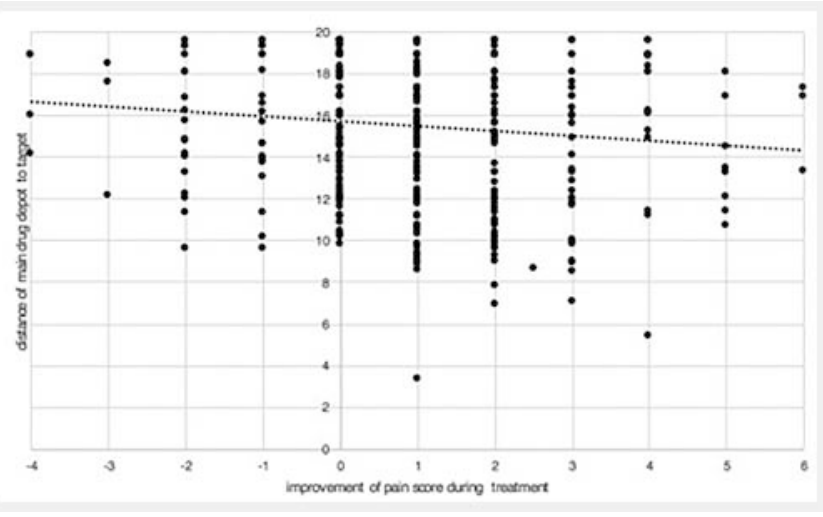

- Fig. 2 Correlation between drug deposit and pain score; there is a weak correlation between short distance of drug deposit and good pain relief.

whereas Physician D was the least successful with a pain reduction of 0.6 points ( $\mathbf{F i g} .4$ ). Pain reduction achieved by Physicians $A$ and $B$ was identical at 0.8 , while technically Physician A achieved a significantly better result than Physician $B$. With an average pain reduction of 0.6 points per treatment, Physician $D$ showed the worst pain improvement values, although technical success was even slightly better than with Physician B.

Physicians $A$ and E exhibited relatively similar medication distribution patterns; the deviation was only $3 \%$, but the reduction of the pain score was $63 \%$ higher for Physician E compared to Physician A (0.8 vs. 1.3). The technically most proficient Physician C still did not quite reach the level of Physician $E$, who had the best result per procedure. With an average improvement in the pain score per intervention of 0.8 , Physicians A and B show exactly the same value, although technically Physician $B$ showed a $17 \%$ greater distance of the drug deposit from the target point than Physician A ( $>$ Fig. 4, 5) ( Table 1 ). No significant correlation could be shown between the absolute needle position and the expected distribution of the drug mixture $(c=-0.068, p<0.0084)$. Likewise, there was no correlation between the radiologist's experience and therapeutic success.

To determine whether the therapeutic outcome correlated with the treating radiologist, we carried out an exact Fischer test in which we first analyzed whether there was a connection between general improvement of symptoms - good or very good improvement - and the treating physician; followed by the same test, which checked whether there was a connection between a very good improvement and the treating physician. As a null hypothesis it was assumed that there is no dependence on the treating physician. In both cases the null hypothesis was ruled out, with $\mathrm{p}<0.0084$ for general improvement (good or very good) and with $p<0.034$ for very good improvement under treatment. This observation demonstrates that, in addition to technical success, the physician performing the procedure plays a decisive role in therapy - either as a person, through his actions or as an effective combination of both.

The pain reduction resulting from the therapy exhibits no detectable difference over time. 


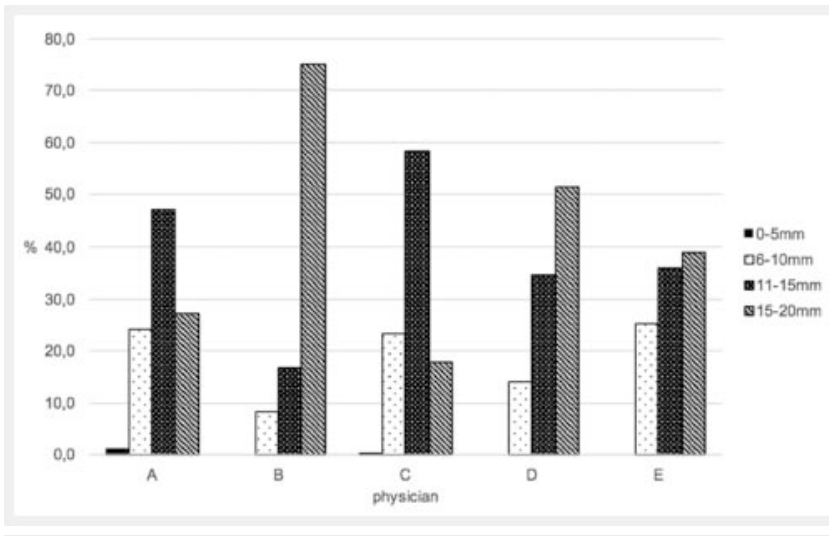

- Fig. 3 Average distance of the drug deposit from the neural foramen by treating physician.

As therapy-normal side effects, $32 \%$ of the treated patients complained of a slight muscular numbness in the corresponding muscle, in $9 \%$ a moderate degree of numbness was reported. However, all patients were able to return to the home environment after treatment.

In one patient, syncope occurred when exiting the CT table due to hypotension immediately after periradicular therapy. After infusion therapy and temporary repositioning to the horizontal position, this patient was completely well again and he could be discharged home. Severe side effects were not observed.

\section{Discussion}

Our study concurred with the literature and showed a very good effectiveness of CT-guided periradicular therapy [17-19].

Overall, $90 \%$ of the patients showed an effective reduction of pain under therapy; $49 \%$ demonstrated a very good improvement of symptoms under therapy and $41 \%$ exhibited a good improvement of symptoms. Similarly, a study by Vad et al. showed a good improvement in symptoms in $84 \%$ of patients treated with transforaminal therapy [20]. In their study, Thomas et al. showed a better outcome in back pain patients when they were treated with transforaminal therapy than when intraspinous medication was applied epidurally [17]. The outcome was better for the transforaminally-treated patients in both short-term and long-term comparisons [17]. A further study by Yang et al. showed that in $63 \%$ of their patients it was possible to avoid a pending operation by employing transforaminal therapy; this was reported in a longterm course over 24 months [18].

A common problem of the studies on back pain is the different perception and evaluation of pain. Despite an explained and defined pain scale, even the same individual evaluates the supposedly same pain differently depending on internal and external factors [21]. In addition, there are differences in pain perception depending on age, gender, ethnic origin as well as previously experienced pain $[22,23]$. Our study design did not provide for a long-term course, so we can only assess short-term success with our data. Study patients whom we saw again in the course of other examinations reported a sustained improvement in symp-

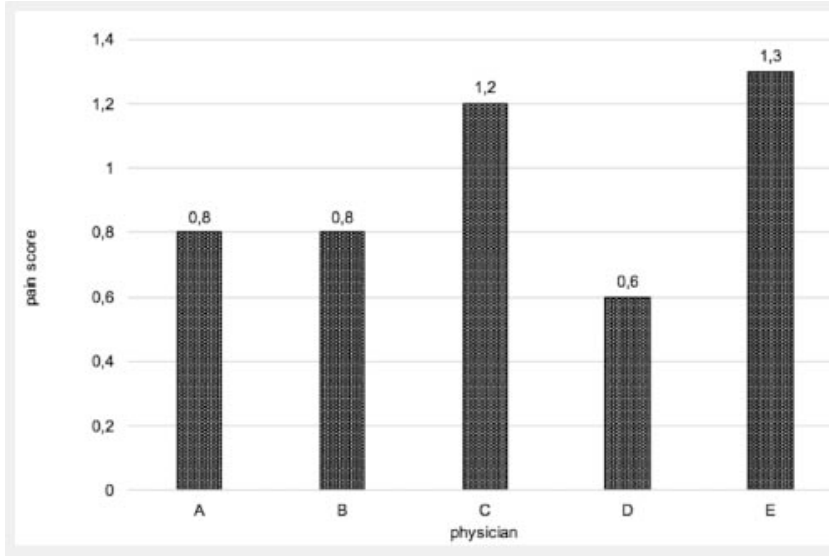

Fig. 4 Average improvement of the pain score per treatment session by treating physician.

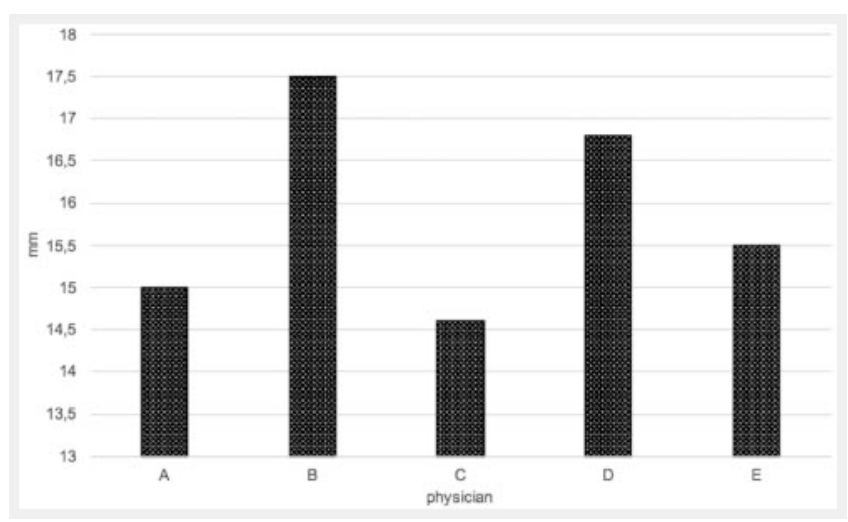

- Fig. 5 Average distance of the drug deposit by treating physician.

toms. However, these individual reports cannot ultimately be evaluated conclusively.

In accordance with the above-mentioned investigations, no therapy-related complications were observed in our patients, so that in agreement with the literature it can be stated that the procedure is effective and has few side effects. The success rates we achieved are better compared to those reported in the literature. This may be due to the referral system in Germany which has changed in recent years. The patients were referred for therapy only after prior consultation with a pain therapist. As a result, patient selection may have changed compared to the above-mentioned studies, since only particularly suitable patients were referred for CT-guided periradicular therapy.

Our data showed that in fact only a small portion of the drug mixture was applied directly around the nerve root. Large amounts of the drug mixture were located in the extraforaminal area. This distribution is ultimately determined by the anatomy of the spinal column and neural foramina. An anatomical study by Teske et al. showed that the anterolateral epidural space of the nerve root in segment L5/S1 is on average only $1.1 \mathrm{ml}$ [19]. Since this epidural area directly around the emerging $L 5$ root is additionally filled with cerebrospinal fluid, the space available in the patient - in contrast to the anatomical preparation - is reduced 
- Table 1 Comparison of the achieved pain reduction and position/distribution of the drug mixture absolutely and relative to the mean value as well as the best value.

\begin{tabular}{|c|c|c|c|c|c|c|}
\hline & pain score & reduction & & $\begin{array}{l}\text { dis- } \\
\text { tance }\end{array}$ & medication & mixture \\
\hline physician & $\begin{array}{l}\text { absolute per } \\
\text { treatment } \\
\text { session }\end{array}$ & $\begin{array}{l}\% \text { compared to } \\
\text { best physician }\end{array}$ & $\begin{array}{l}\% \text { compared to } \\
\text { mean of all } \\
\text { physicians }\end{array}$ & in $\mathrm{mm}$ & $\begin{array}{l}\% \text { compared to } \\
\text { best physician }\end{array}$ & $\begin{array}{l}\text { \% compared to } \\
\text { mean of all } \\
\text { physicians }\end{array}$ \\
\hline A & 0.8 & -38.5 & 17.5 & 15 & 2.7 & 5.6 \\
\hline B & 0.8 & -38.5 & 17.5 & 17.5 & 19.9 & -9.5 \\
\hline C & 1.2 & -7.7 & -21.7 & 14.6 & 0 & 8.5 \\
\hline D & 0.6 & -53.8 & 56.7 & 16.8 & 15.1 & -5.7 \\
\hline E & 1.3 & 0 & -27.7 & 15.3 & 4.8 & 3.5 \\
\hline best value & 1.3 & & & 14.6 & & \\
\hline mean & 0.9 & & & 15.8 & & \\
\hline
\end{tabular}

even further [19]. The distribution space we have documented is therefore reasonable due to anatomical conditions; the lack of correlation between the needle position and the distribution of the medication mixture can also be explained by anatomical conditions. The volume of perineural and intraforaminal distribution as well as after intraspinous epidural application is limited compared to the extraforaminal distribution volume, which is why the largest amounts of the drug mixture are located extraforaminally. Distribution cannot be predicted, since the epidural space around the nerve root is additionally configured asymmetrically and has a pronounced interindividual characteristic. Even a supposedly extraforaminal needle position can lead to a relatively pronounced epidural deposit of the drug mixture, whereas even with a safe intraforaminal position of the needle, the drug mixture is distributed extraforaminally in large parts. Likewise, an intramuscular distribution in parts or a distribution along the extraforaminal fascia is by no means unusual despite an optimal needle position. These anatomical variants were also shown in a further study, in which it was checked whether the drug mixture was accidentally injected into the vascular system as part of periradicular therapy. Intravascular distribution of the medication applied is evident in $8-9 \%$ of cases. The risk of this intravascular misinjection was shown in this study to be significantly lower if the needle position was slightly extraforaminally positioned [24].

This shows that a safe and yet effective needle position for the patient should be sought, especially since an absolute needle position is not correlated with success.

Our data show that there is a significant correlation between the treating physician and the success of the therapy. Further studies should be carried out to determine whether this is due to the physician performing the treatment as a person or to his behavior or to some extent both. It is crucial to note that there is a side-effect-free, doctor-specific component that has an influence on the therapy outcome. This connection has not yet been established and examined in the literature.

\section{Summary}

In addition to a good technical implementation of the CT-guided periradicular therapy, the doctor performing the intervention is significantly involved in the success of the therapy. The therapy, based on a good and carefully balanced clinical indication, shows a good improvement in symptoms in $90 \%$ of the cases without any noteworthy complications.

\section{Conflict of Interest}

Die Firma Dr. Ausbüttel und Co. GmbH hat die Stelle eines wissenschaftlichen Mitarbeiters (Benedikt Hackert) an der Universität Witten/Herdecke finanziert, der freundlicher Weise unser Projekt unterstützen durfte. Produkte der Firma Dr. Ausbüttel wurden nicht untersucht oder verwendet, es wurde keinerlei Einflussnahme auf das Projekt genommen. Die Förderung war direkt an die Universität selbst gerichtet.

\section{References}

[1] Manchikanti L, Singh V, Falco FJ et al. Epidemiology of low back pain in adults. Neuromodulation: journal of the International Neuromodulation Society 2014; 17 (Suppl. 23): 10

[2] Geneen L], Moore RA, Clarke C et al. Physical activity and exercise for chronic pain in adults: an overview of Cochrane Reviews. The Cochrane database of systematic reviews 2017; 4CD011279

[3] Kuijpers T, van Middelkoop M, Rubinstein SM et al. A systematic review on the effectiveness of pharmacological interventions for chronic nonspecific low-back pain. European spine journal: official publication of the European Spine Society, the European Spinal Deformity Society, and the European Section of the Cervical Spine Research Society 2011; 20: 4050

[4] Breivik H, Eisenberg E, O'Brien T et al. The individual and societal burden of chronic pain in Europe: the case for strategic prioritisation and action to improve knowledge and availability of appropriate care. BMC public health 2013; 131229 
[5] Hoy D, Brooks P, Blyth F et al. The Epidemiology of low back pain. Best practice \& research Clinical rheumatology 2010; 24: 769-781

[6] Jacobs WC, van Tulder M, Arts M et al. Surgery versus conservative management of sciatica due to a lumbar herniated disc: a systematic review. European spine journal: official publication of the European Spine Society, the European Spinal Deformity Society, and the European Section of the Cervical Spine Research Society 2011; 20: 513-522

[7] Manchikanti L, Singh V, Cash KA et al. Effect of fluoroscopically guided caudal epidural steroid or local anesthetic injections in the treatment of lumbar disc herniation and radiculitis: a randomized, controlled, double blind trial with a two-year follow-up. Pain physician 2012; 15: 273-286

[8] Lee JW, Kim SH, Choi JY et al. Transforaminal epidural steroid injection for lumbosacral radiculopathy: preganglionic versus conventional approach. Korean journal of radiology 2006; 7: 139-144

[9] Waggershauser T, Schwarzkopf S, Reiser M. Facet blockade, peridural and periradicular pain therapy. Der Radiologe 2006; 46: 520-526. Facettenblockade, peridurale und periradikulare Schmerztherapie

[10] Buttermann GR. Treatment of lumbar disc herniation: epidural steroid injection compared with discectomy. A prospective, randomized study. The Journal of bone and joint surgery American volume 2004; 86-A: 670-679

[11] Kaye AD, Manchikanti L, Abdi S et al. Efficacy of Epidural Injections in Managing Chronic Spinal Pain: A Best Evidence Synthesis. Pain physician 2015; 18: E939-E1004

[12] Manchikanti L, Knezevic NN, Boswell MV et al. Epidural Injections for Lumbar Radiculopathy and Spinal Stenosis: A Comparative Systematic Review and Meta-Analysis. Pain physician 2016; 19: E365-E410

[13] Wewalka M, Abdelrahimsai A, Wiesinger GF et al. CT-guided transforaminal epidural injections with local anesthetic, steroid, and tramadol for the treatment of persistent lumbar radicular pain. Pain physician 2012; 15: 153-159

[14] Maurer MH, Schreiter N, de Bucourt M et al. Cost comparison of nerve root infiltration of the lumbar spine under MRI and CT guidance. European radiology 2013; 23: 1487-1494

[15] Manchikanti L, Pampati V, Benyamin RM et al. Cost Utility Analysis of Lumbar Interlaminar Epidural Injections in the Treatment of Lumbar Disc Herniation, Central Spinal Stenosis, and Axial or Discogenic Low Back Pain. Pain physician 2017; 20: 219-228
[16] Lee JH, Shin KH, Bahk S] et al. Comparison of clinical efficacy of transforaminal and caudal epidural steroid injection in lumbar and lumbosacral disc herniation: A systematic review and meta-analysis. The spine journal: official journal of the North American Spine Society 2018

[17] Thomas E, Cyteval C, Abiad L et al. Efficacy of transforaminal versus interspinous corticosteroid injectionin discal radiculalgia - a prospective, randomised, double-blind study. Clinical rheumatology 2003; 22: 299304

[18] Yang SC, Fu TS, Lai PL et al. Transforaminal epidural steroid injection for discectomy candidates: an outcome study with a minimum of two-year follow-up. Chang Gung medical journal 2006; 29: 93-99

[19] Teske W, Zirke S, Nottenkamper J et al. Anatomical and surgical study of volume determination of the anterolateral epidural space nerve root L5/S1 under the aspect of epidural perineural injection in minimal invasive treatment of lumbar nerve root compression. European spine journal: official publication of the European Spine Society, the European Spinal Deformity Society, and the European Section of the Cervical Spine Research Society 2011; 20: 537-541

[20] Vad VB, Bhat AL, Lutz GE et al. Transforaminal epidural steroid injections in lumbosacral radiculopathy: a prospective randomized study. Spine 2002; 27: 11-16

[21] Mclver TA, Kornelsen J, Stroman PW. Diversity in the emotional modulation of pain perception: An account of individual variability. European journal of pain 2018; 22: 319-332

[22] Lavin R, Park J. A characterization of pain in racially and ethnically diverse older adults: a review of the literature. Journal of applied gerontology: the official journal of the Southern Gerontological Society 2014; 33: 258-290

[23] Park J, Newman D, Engstrom G et al. The moderating and covarying effects of social support and pain intensity on depressive symptomology among racially and ethnically diverse older adults. Pain management 2017; 7: 19-32

[24] Yu RK, Lagemann GM, Ghodadra A et al. Extraforaminal needle tip position reduces risk of intravascular injection in CT-fluoroscopic lumbar transforaminal epidural steroid injections. Journal of spine surgery 2016; 2: $246-255$ 